\title{
Self-improving properties of weighted Gehring classes with applications to partial differential equations
}

\author{
S.H. Saker ${ }^{1,2}$, J. Alzabut ${ }^{3,4^{*}}$ (D, D. O'Regan ${ }^{5}$ and R.P. Agarwal ${ }^{6}$
}

\section{"Correspondence:}

jalzabut@psu.edu.sa

${ }^{3}$ Department of Mathematics and General Sciences, Prince Sultan University, 11586 Riyadh, Saudi Arabia

${ }^{4}$ Department of Industrial Engineering, OSTIM Technical University, 06374 Ankara, Turkey Full list of author information is available at the end of the article

\begin{abstract}
In this paper, we prove that the self-improving property of the weighted Gehring class $G_{\lambda}^{p}$ with a weight $\lambda$ holds in the non-homogeneous spaces. The results give sharp bounds of exponents and will be used to obtain the self-improving property of the Muckenhoupt class $A^{9}$. By using the rearrangement (nonincreasing rearrangement) of the functions and applying the Jensen inequality, we show that the results cover the cases of non-monotonic functions. For applications, we prove a higher integrability theorem and report that the solutions of partial differential equations can be solved in an extended space by using the self-improving property. Our approach in this paper is different from the ones used before and is based on proving some new inequalities of Hardy type designed for this purpose.
\end{abstract}

MSC: Primary 26D15; 34A40; secondary 34N05; 39A12

Keywords: Reverse Hölder's inequality; Muckenhoupt type inequality; Reverse Hölder's inequality; Higher integrability; Gehring type inequalities

\section{Introduction}

Let $I_{0}$ be a fixed interval of $\mathbb{R}$, and by $|I|$ for any arbitrarily interval $I \subset I_{0}$, we mean its Lebesgue measure. We say that $u$ is a weight if it is a locally integrable function on the real line, positive almost everywhere (with respect to the Lebesgue measure). The weight $u$ belongs to the Muckenhoupt class $A^{s}$ for $1<s<\infty$ if there exists a constant $\mathcal{C}>1$ such that

$$
\left(\frac{1}{|I|} \int_{I} u(t) d t\right)\left(\frac{1}{|I|} \int_{I} u^{-\frac{1}{s-1}}(t) d t\right)^{s-1} \leq \mathcal{C} .
$$

The $A^{s}$-constant of $u$ is

$$
A^{s}(u):=\sup _{I}\left(\frac{1}{|I|} \int_{I} u(t) d t\right)\left(\frac{1}{|I|} \int_{I} u^{-\frac{1}{s-1}}(t) d t\right)^{s-1},
$$

where the supremum is taken over all $I \subset I_{0}$. For a given fixed constant $\mathcal{C}>1$, if the weight $u$ belongs to $A^{s}(\mathcal{C})$, then $A^{s}(u) \leq \mathcal{C}$. The $A^{s}$ class of weights was introduced by Muckenhoupt

(c) The Author(s) 2021. This article is licensed under a Creative Commons Attribution 4.0 International License, which permits use, sharing, adaptation, distribution and reproduction in any medium or format, as long as you give appropriate credit to the original author(s) and the source, provide a link to the Creative Commons licence, and indicate if changes were made. The images or other third party material in this article are included in the article's Creative Commons licence, unless indicated otherwise in a credit line to the material. If material is not included in the article's Creative Commons licence and your intended use is not permitted by statutory regulation or exceeds the permitted use, you will need to obtain permission directly from the copyright holder. To view a copy of this licence, visit http://creativecommons.org/licenses/by/4.0/. 
in [21] and was used to prove the boundedness of the Hardy-Littlewood maximal operator

$$
M f(x):=\sup _{x \in I} \frac{1}{|I|} \int_{I}|f(y)| d y
$$

in the class $L_{u}^{s}\left(\mathbb{R}_{+}\right)$with a weight $u$. In fact it was proved that if $1<s<\infty$, then $M f$ is bounded in $L_{u}^{s}\left(\mathbb{R}_{+}\right)$if and only if $u \in A^{s}$. Hunt, Muckenhoupt, and Wheeden [12] also gave a characterization for the weights in the Hilbert operator and proved that the precise condition of the boundedness of Hilbert's operator is the $A^{s}$-condition (1). Note that from Hölder's inequality $A^{s}(u) \geq 1$ for all $1<s<\infty$ and the following inclusion is true:

$$
\text { if } 1<s<r<\infty \text {, then } A^{s} \subset A^{r} \text { and } A^{r}(u) \leq A^{s}(u) \text {. }
$$

Muckenhoupt [21] and Coifman and Fefferman [20] proved that if $u$ satisfies (1) then there exist $r<s$ and a positive constant $\mathcal{C}_{1}$ such that

$$
\left(\frac{1}{|I|} \int_{I} u(t) d t\right)\left(\frac{1}{|I|} \int_{I} u^{-\frac{1}{r-1}}(t) d t\right)^{r-1} \leq \mathcal{C}_{1} \quad \text { for all } I \subset I_{0}
$$

i.e., Muckenhoupt and Coifman and Fefferman's result for self-improving property states that: if $u \in A^{s}(\mathcal{C})$ then there exist a constant $\epsilon>0$ and a positive constant $\mathcal{C}_{1}$ such that $u \in A^{s-\epsilon}\left(\mathcal{C}_{1}\right)$, and then

$$
A^{s}(\mathcal{C}) \subset A^{s-\epsilon}\left(\mathcal{C}_{1}\right) \text {. }
$$

A weight $u$ satisfies the $A^{1}$-Muckenhoupt condition if there exists a constant $\mathcal{C} \geq 1$ such that, for any arbitrary interval $I \subset I_{0}$, one has

$$
M f(x) \leq \mathcal{C} u(x) \text { for all } x \in I
$$

In [21] it was proved that if (4) holds, then for every $s \in[1, \mathcal{C} /(\mathcal{C}-1)]($ here $\mathcal{C}>1)$, the function $u$ satisfies reverse Hölder's inequality

$$
\frac{1}{|I|} \int_{I} u^{s}(x) d x \leq C_{s}\left(\frac{1}{|I|} \int_{I} u(x) d x\right)^{s}, \quad \text { where } C_{s}=\frac{\mathcal{C}}{\mathcal{C}-s(\mathcal{C}-1)}
$$

The constant $C_{s}$ in (5) was improved by Bojarski et al. in [3] and replaced with $C_{s}=$ $\mathcal{C}^{1-s} /(\mathcal{C}-s(\mathcal{C}-1))$. Another important class of weights, the $G^{r}$-class for $1<r<\infty$, was introduced by Gehring $[10,11]$ in connection with local integrability properties of the gradient of quasiconformal mappings. A weight $u$ satisfies the $G^{r}$ condition (or is said to belong to $G^{r}(\mathcal{K})$ ) if there exists $\mathcal{K}>1$ such that

$$
\left(\frac{1}{|I|} \int_{I} u^{r}(x) d x\right)^{1 / r} \leq \mathcal{K}\left(\frac{1}{|I|} \int_{I} u(x) d x\right) \quad \text { for all } I \subset I_{0}
$$

The smallest constant $\mathcal{K}$ verifying (6) is called the $G^{r}$-norm of the weight $u$ and is denoted by $G^{r}(u)$ and given by

$$
G^{r}(u)=\sup _{I}\left(\frac{\left(\frac{1}{|I|} \int_{I} u^{r}(x) d x\right)^{1 / r}}{\frac{1}{|I|} \int_{I} u(x) d x}\right)^{r /(r-1)},
$$


where the supremum is taken over all $I \subset I_{0}$. For a given fixed constant $\mathcal{K}>1$, if the weight $u$ belongs to $G^{r}(\mathcal{K})$, then $G^{r}(u) \leq \mathcal{K}$. Note that from Hölder's inequality $G^{r}(v) \geq 1$ for all $1<r<\infty$ and the following inclusion is true:

$$
\text { if } 1<s<r<\infty \text {, then } G^{r} \subset G^{s} \text { and } 1 \leq G^{s}(v) \leq G^{r}(v) \text {. }
$$

Gehring proved that if (6) holds, then there exist $s>r$ and a positive constant $\mathcal{K}_{1}$ such that

$$
\frac{1}{|I|} \int_{I} u^{s}(x) d x \leq \mathcal{K}_{1}\left(\frac{1}{|I|} \int_{I} u(x) d x\right)^{s}
$$

In other words, Gehring's result for the self-improving property states that: if $u \in G^{r}(\mathcal{K})$ then there exist $\epsilon>0$ and a positive constant $\mathcal{K}_{1}$ such that $u \in G^{r+\epsilon}\left(\mathcal{K}_{1}\right)$, and then

$$
G^{r}(\mathcal{K}) \subset G^{r+\epsilon}\left(\mathcal{K}_{1}\right) .
$$

Researchers (for (3) see $[1,7-9,15-17,22,24]$, for (8) see $[2,6,15,23,27,29-32]$ ) were interested in:

$\left(h_{1}\right)$ Finding the exact value of the limit exponent (the value of $\epsilon$ ) for which the selfimproving properties hold;

$\left(h_{2}\right)$ Finding the best constants $\mathcal{C}_{1}$ and $\mathcal{K}_{1}$.

Korenovskii [15] found the sharp lower bound of the exponent (self-improving property) for which (3) holds and proved that the optimal integrability exponent $r^{*}$ is the positive root of the equation

$$
\frac{1}{x}\left(\frac{r-1}{r-x}\right)^{r-1}=\mathcal{C}
$$

and the exact value of $\epsilon$ is given by $r-r^{*}$, and the author also found an explicit value of the constant of the new class. D'Apuzzo and Sbordone [6] found the optimal integrability exponent for monotonic $G^{r}$ weights as a solution of an algebraic equation

$$
\left(\frac{x}{x-1}\right)^{-1}\left(\frac{x}{x-r}\right)^{\frac{1}{r}}=\mathcal{K}
$$

The relations between Gehring and Muckenhoupt classes (inclusions properties) were given by Coifman and Fefferman [4]. They proved that any Gehring class is contained in some Muckenhoupt class, and vice versa. In other words, the inclusions

$$
G^{r}(\mathcal{K}) \subset A^{s}\left(\mathcal{K}_{1}\right)
$$

and

$$
A^{s_{1}}\left(\mathcal{K}_{1}\right) \subset G^{r_{1}}(\mathcal{K})
$$

hold. The sharp bound of exponents for which inclusion (12) is valid was obtained by Bojarski et al. [3] when $s_{1}=1$. In fact their result (inclusion property) proves that if $u \in A^{1}$ 
with $A^{1}(u)=\mathcal{C}$, then $u \in G^{s}$ for every $1 \leq s<\mathcal{C} /(\mathcal{C}-1)$ with a constant

$$
G^{s}(u) \leq\left(\frac{\mathcal{C}^{1-s}}{\mathcal{C}-s(\mathcal{C}-1)}\right)^{1 /(s-1)} .
$$

The constant on the right-hand side as well as the upper bounds of $s$ cannot be improved. In fact the weight $u(t)=t \frac{1}{\mathcal{C}-1} / \mathcal{C}$ is an extremal, which gives equality in (13) and lies in $L^{s}$ if and only if $s<\mathcal{C} /(\mathcal{C}-1)$. The sharp bounds of exponents for which inclusion (11) is valid were obtained in [18], and the sharp bounds of exponents for which inclusion (12) is valid were obtained in [19]. In [26, 28] the authors employed Hardy and Hardy-Littlewood type inequalities and proved that the constant of the new class satisfies

$$
\left[G^{S}(u)\right]^{1 / s^{\prime}} \leq \mathcal{K}^{1 / r^{\prime}}\left(\frac{r}{s \mathcal{K}(s)}\right)^{1 / r}
$$

where

$$
\mathcal{K}(s)=1-\mathcal{K}^{r} \frac{(s-r)}{s}\left(\frac{s}{s-1}\right)^{r} .
$$

To illustrate the interest of the $G^{s}$ class, Pérez [25] proved that the weighted operator

$$
M_{u} f(x):=\sup _{x \in I} \frac{1}{u(I)} \int_{I}|f(y)| u(y) d y
$$

is bounded in $L_{u}^{s}\left(\mathbb{R}_{+}\right)$if and only if $u \in G^{s^{\prime}}$. Further, Vasyunin [33] and Dindos and Wall [7] found the sharp constants respectively for Muckenhoupt and Gehring weights by using the powerful technique of the Bellman functions. In [27], Popoli obtained these results for a larger class of weights verifying a more general reverse Hölder inequality (hence for Muckenhoupt and Gehring weights as particular cases). In [31,32] Sbordone considered a new class $G_{\omega}^{r}$ of Gehring type with weights and proved that the self-improving property of this class also holds. We say that the nonnegative measurable function $u$ satisfies the weighted Gehring $G_{\omega}^{r}$-condition if there exists a constant $\mathcal{K} \geq 1$ such that

$$
\left(\frac{1}{W(I)} \int_{I} u^{r}(x) \omega(x) d x\right)^{1 / r} \leq \mathcal{K}\left(\frac{1}{W(I)} \int_{I} \omega(x) u(x) d x\right)
$$

for all $I \subset I_{0}$, where $W(I)=\int_{I} \omega(x) d x$. The $G_{\omega}^{r}$-constant of $u$ is

$$
G_{\omega}^{r}(u)=\sup _{I}\left(\frac{\left(\frac{1}{W(I)} \int_{I} \omega(x) u^{r}(x) d x\right)^{1 / r}}{\frac{1}{W(I)} \int_{I} \omega(x) u(x) d x}\right)^{r /(r-1)}
$$

for all $I \subset I_{0}$. One can see that if $u \in A^{s}(\mathcal{C})$ then condition (1) holds and can be rewritten in the form

$$
\left(\frac{1}{W(I)} \int_{I}\left(\frac{1}{u(t)}\right)^{\bar{s}} u(t) d t\right)^{\frac{1}{s}} \leq \mathcal{K}\left(\frac{1}{W(I)} \int_{I}\left(\frac{1}{u(t)}\right) u(t) d t\right)
$$

where $W(I)=\int_{I} u(t) d t$ and $\bar{s}=s /(s-1)$, which is a weighted $G_{u}^{\bar{s}}(\mathcal{K})$ condition for $u^{-1}$ with respect to the weight $u$. This shows that if $u \in A^{s}(\mathcal{C})$ then $u^{-1} \in G_{u}^{\bar{s}}(\mathcal{K})$ with $\mathcal{K}=\mathcal{C}^{\frac{\bar{s}-1}{\bar{s}}}$. In 
[32, Theorem 4.1] Sbordone proved that if (16) holds and $u(x) d x$ is a doubling measure, i.e., there exists a constant $d>0$ such that

$$
W(2 I) \leq d W(I)
$$

then there exists $s>r$ such that

$$
\left(\frac{1}{W(I)} \int_{I} u^{s}(x) \omega(x) d x\right)^{1 / s} \leq \mathcal{K}_{1}\left(\frac{1}{W(I)} \int_{I} \omega(x) u(x) d x\right)
$$

In [32] Sbordone used the self-improving property of the class $G_{\omega}^{r}$ and proved that the backward propagation of the class $A^{s}$ holds and proved $A^{s}(\mathcal{C}) \subset A^{s-\epsilon}\left(\mathcal{C}_{1}\right)$ without the lower bounds of the constant $\epsilon$. The space of functions that satisfy condition (18) is called the spaces of homogeneous type. Many results from real and harmonic analysis on Euclidean spaces have their natural extensions on these spaces. Another key tool in studying the reverse Hölder inequalities are the equimeasurable properties of monotonic rearrangements and their applications in extending such results to the $n$-dimensional case that has been applied for the first time by Sbordone in [32] and refined in [8, 9, 15, 17, 30, 31]. Our aim in this paper is to study the structure of the weighted Gehring classes and also prove that the self- improving properties hold with exact values of the exponents and the constants of transitions. Our results will be proved in the nonhomogeneous spaces, i.e., when (18) does not hold.

The paper is organized as follows: In Sect. 2, first we prove some new weighted refinement inequalities of Hardy type. Second, we study the structure of the weighted Gehring classes and also prove that the self-improving properties hold with exact values of the exponents and the constants of transitions. The technique in this paper allows us to give an improvement of the constants in the classes. We also prove that if $u \in A_{\omega}^{1}(\mathcal{C})$ then there exists $s>1$ such that $u \in G_{\omega}^{s}\left(\mathcal{C}_{1}\right)$ with a sharp constant $\mathcal{C}_{1}$ similar to the constant obtained by Bojarski et al. [3]. We establish sharp bounds of exponents for which inclusion (3) is valid from the self-improving property $G_{\omega}^{s}(\mathcal{K})$, and we also prove a higher integrability theorem by applying the inclusion property between $A_{\omega}^{1}(\mathcal{C})$ and $G_{\omega}^{s}(\mathcal{K})$. In Sect. 3, we recall some applications which show the interest of our main results.

\section{Main results}

Throughout the paper, we assume (usually without mentioning) that the functions in the statements of the theorems are nonnegative and integrable, and the integrals considered are assumed to exist. We fix an interval $I_{0} \subset \mathbb{R}_{+}=[0, \infty)$ and consider subintervals $I$ of $I_{0}$ of the form $[a, s]$ for $a<s<\infty$ (or $[a, \infty)$ ) and assume that $\omega$ is a positive integrable function defined on $I_{0}$; here $a \geq 0$. For simplicity, we set

$$
\begin{aligned}
& W(t)=\int_{a}^{t} \omega(x) d x \\
& A(t)=\int_{a}^{t} \omega(x) u(x) d x \quad \text { for } t \in I, \text { where } I=[a, \cdot] \text { or }[a, \infty)
\end{aligned}
$$


For any weight $u$ which is a nonnegative integrable function, we define the operator $\mathcal{H} u$ : $I \rightarrow \mathbb{R}^{+}$by

$$
\mathcal{H} u(t)=\frac{1}{W(t)} \int_{a}^{t} \omega(x) u(x) d x \quad \text { for all } t \in I
$$

From the definition of $\mathcal{H}$, we see that if $u$ is nondecreasing, then

$$
\mathcal{H} u(t)=\frac{1}{W(t)} \int_{a}^{t} \omega(x) u(x) d x \leq \frac{1}{W(t)} \int_{a}^{t} \omega(x) u(t) d x=u(t)
$$

Also, using the above inequality, we have that

$$
(\mathcal{H} u(t))^{\prime}=\frac{1}{W(t)}[u(t)-\mathcal{H} u(t)] \geq 0 \quad \text { for } t \in I .
$$

From these two facts, we have the following properties of $\mathcal{H} u$ for nondecreasing functions.

\section{Lemma 2.1}

(i) If $u$ is a nondecreasing function, then $\mathcal{H} u(t) \leq u(t)$.

(ii) If $u$ is a nondecreasing function, then so is $\mathcal{H u}$.

Remark 2.1 As a consequence of Lemma 2.1, if $r>1$ we deduce that $\mathcal{H} u^{r} \leq u^{r}$ when $u$ is a nondecreasing function. We also notice from Lemma 2.1 that if $u$ is nondecreasing, then $\mathcal{H} u^{r}$ is also nondecreasing.

Also, from the definition of $\mathcal{H}$, we see that if $u$ is nonincreasing, then

$$
\mathcal{H} u(t)=\frac{1}{W(t)} \int_{a}^{t} \omega(x) u(x) d x \geq \frac{1}{W(t)} \int_{a}^{t} \omega(x) u(t) d x=u(t) .
$$

Also, by using the above inequality, we have that

$$
(\mathcal{H} u(t))^{\prime}=\frac{1}{W(t)}[u(t)-\mathcal{H} u(t)] \leq 0 \quad \text { for } t \in I .
$$

From these two facts, we have the following properties of $\mathcal{H} u$ for nonincreasing functions.

\section{Lemma 2.2}

(i) If $u$ is nonincreasing, then $\mathcal{H} u(t) \geq u(t)$.

(ii) If $u$ is nonincreasing, then so is $\mathcal{H u}$.

Remark 2.2 As a consequence of Lemma 2.2, if $r>1$ then $\mathcal{H} u^{r} \geq u^{r}$ when $u$ is a nonincreasing function. We also notice from Lemma 2.2 that if $u$ is nonincreasing, then $\mathcal{H} u^{r}$ is also nonincreasing.

We need the following lemma in the proofs. 
Lemma 2.3 Let $s \geq r>1$, and define

$$
\Omega(\rho)=\rho-\left(\frac{s}{s-1}\right)^{r} \rho^{-r+1}\left(\frac{s-1}{s} \rho+\frac{1}{s} \rho^{s}\right)^{r}, \quad \text { where } \rho \text { and } \rho>0 \text {. }
$$

Then $\Omega(\rho)$ is an increasing function for $\rho>0$ and $\Omega(\rho) \leq \frac{-r}{s-1} \rho^{s}$.

Proof From the definition of $\Omega$, we see that

$$
\Omega^{\prime}(\rho)=1+(r-1)\left(1+\frac{\rho^{s}}{(s-1) \rho}\right)^{r}-r\left(1+\frac{\rho^{s}}{(s-1) \rho}\right)^{r-1} .
$$

Now, consider the function

$$
\digamma(\tau)=1+(r-1) \tau^{r}-r \tau^{r-1} \quad \text { for } \tau \geq 1 .
$$

It is clear that $\digamma^{\prime}(\tau)>0$ for every $\tau>1$, and thus $\digamma$ is strictly increasing for $\tau \geq 1$ and $\digamma(\tau)>\digamma(1)=0$ for any $\tau>1$. Thus $\Omega^{\prime}(\rho)>0$ for $\rho>0$, so $\Omega(\rho)$ is strictly increasing for $\rho \in(0, \infty)$. From the definition of $\Omega(\rho)$, we see from L'Hospital's rule that

$$
\begin{aligned}
\lim _{\rho \rightarrow \infty} \Omega(\rho) & =\lim _{\rho \rightarrow \infty} \rho\left[1-\left(1+\frac{\rho^{s}}{(s-1) \rho}\right)^{r}\right] \\
& =\lim _{y \rightarrow 0} \frac{1-\left(1+\frac{\rho^{s} y}{(s-1)}\right)^{r}}{y}=-\frac{r}{s-1} \rho^{s} .
\end{aligned}
$$

As a result, $\Omega(\rho) \leq-\frac{r}{s-1} \rho^{s}$ for any $\rho>0$. The proof is complete.

Theorem 2.4 Let $u$ be a nonnegative weight and $A(t)$ and $W(t)$ be defined as in (20). If $s>1$, then

$$
\int_{a}^{\infty} \omega(t)\left(\frac{A(t)}{W(t)}\right)^{s} d t \leq\left(\frac{s}{s-1}\right)^{s} \int_{a}^{\infty} \omega(t) u^{s}(t) d t
$$

Proof Let $x>a$. From (20), we have (here $t \in(a, x))$

$$
\omega(t) u(t)=[W(t) \alpha(t)]^{\prime}, \quad \text { where } \alpha(t)=\frac{A(t)}{W(t)} .
$$

Then

$$
\omega(t) u(t)=\left[W(t) \alpha^{\prime}(t)+\alpha(t) \omega(t)\right]
$$

Now, using (23), we have that

$$
\begin{aligned}
& \omega(t) \alpha^{s}(t)-\frac{s}{s-1} \omega(t) u(t) \alpha^{s-1}(t) \\
& \quad=\omega(t) \alpha^{s}(t)-\frac{s}{s-1} \alpha^{s-1}(t)\left[W(t) \alpha^{\prime}(t)+\alpha(t) \omega(t)\right] \\
& \quad=\omega(t) \alpha^{s}(t)-\frac{s}{s-1} \omega(t) \alpha^{s}(t)-\frac{s}{s-1} W(t) \alpha^{s-1}(t) \alpha^{\prime}(t)
\end{aligned}
$$




$$
\begin{aligned}
& =-\frac{1}{s-1} \omega(t) \alpha^{s}(t)-\frac{s}{s-1} W(t) \alpha^{s-1}(t) \alpha^{\prime}(t) \\
& =-\frac{1}{s-1}\left[\omega(t) \alpha^{s}(t)+s W(t) \alpha^{s-1}(t) \alpha^{\prime}(t)\right]=-\frac{1}{s-1}\left(W(t) \alpha^{s}(t)\right)^{\prime}
\end{aligned}
$$

This leads to

$$
\omega(t) \alpha^{s}(t)-\frac{s}{s-1} \omega(t) u(t) \alpha^{s-1}(t)=-\frac{1}{s-1}\left(W(t) \alpha^{s}(t)\right)^{\prime}
$$

Integrating both sides from $a$ to $x$, we obtain

$$
\int_{a}^{x} \omega(t) \alpha^{s}(t) d t-\frac{s}{s-1} \int_{a}^{x} \omega(t) u(t) \alpha^{s-1}(t) d t=-\frac{1}{s-1} W(x)\left(\alpha^{s}(x)\right)
$$

which leads to

$$
\begin{aligned}
\int_{a}^{x} \omega(t) \alpha^{s}(t) d t \leq & \frac{s}{s-1} \int_{a}^{x} \omega(t) u(t) \alpha^{s-1}(t) d t=\frac{s}{s-1} \int_{a}^{x} \omega^{\frac{1}{s}}(t) u(t) \\
& \times \omega^{\frac{s}{s-1}}(t) \alpha^{s-1}(t) d t
\end{aligned}
$$

Applying Hölder's inequality on the right-hand side with indices $s$ and $s /(s-1)$, we have

$$
\int_{a}^{x} \omega(t) \alpha^{s}(t) d t \leq \frac{s}{s-1}\left\{\int_{a}^{x} \omega(t)\left(u^{s}(t)\right) d t\right\}^{\frac{1}{s}}\left\{\int_{a}^{x} \omega(t) \alpha^{s}(t) d t\right\}^{\frac{s-1}{s}}
$$

Thus

$$
\int_{a}^{x} \omega(t) \alpha^{s}(t) d t \leq\left(\frac{s}{s-1}\right)^{s} \int_{a}^{x} \omega(t)\left(u^{s}(t)\right) d t
$$

which can be written as

$$
\int_{a}^{x} \omega(t)\left(\frac{A(t)}{W(t)}\right)^{s} d t \leq\left(\frac{s}{s-1}\right)^{s} \int_{a}^{x} \omega(t)\left(u^{s}(t)\right) d t
$$

Let $x \rightarrow \infty$ and we obtain

$$
\int_{a}^{\infty} \omega(t)\left(\frac{A(t)}{W(t)}\right)^{s} d t \leq\left(\frac{s}{s-1}\right)^{s} \int_{a}^{\infty} \omega(t)\left(u^{s}(t)\right) d t
$$

which is (22). This completes the proof.

From Theorem 2.4 (see (25) for the second result), we get the following results in terms of $\mathcal{H} u$.

Lemma 2.5 Let $u$ be a nonnegative weight, and let $\mathcal{H}$ be defined as in (21). If $s>1$, then (here $t \in I$ )

$$
\mathcal{H}(\mathcal{H} u)^{s}(t) \leq\left(\frac{s}{s-1}\right)^{s}\left(\mathcal{H} u^{s}\right)(t)
$$


Lemma 2.6 Let $u$ be a nonnegative weight, and let $\mathcal{H}$ be defined as in (21). If $s>1$, then (here $x \in I$ )

$$
\begin{aligned}
\frac{1}{W(x)} \int_{a}^{x} \omega(t)(\mathcal{H} u(t))^{s} d t= & \frac{s}{s-1} \frac{1}{W(x)} \int_{a}^{x} \omega(t) u(t)(\mathcal{H} u(t))^{s-1} d t \\
& -\frac{1}{s-1}(\mathcal{H} u(x))^{s} .
\end{aligned}
$$

As a consequence of Lemma 2.6, if we replace $s$ with $s / r>1$ and $u$ with $u^{r}$, we get the following result.

Lemma 2.7 Let $u$ be a nonnegative weight, and let $\mathcal{H}$ be defined as in (21). If $s \geq r>1$, then (here $x \in I$ )

$$
\begin{aligned}
\frac{1}{W(x)} \int_{a}^{x} \omega(t)\left(\mathcal{H} u^{r}(t)\right)^{s / r} d t= & \frac{s}{s-r} \frac{1}{W(x)} \int_{a}^{x} \omega(t) u^{r}(t)\left(\mathcal{H} u^{r}(t)\right)^{\frac{s}{r}-1} d t \\
& -\frac{r}{s-r}\left(\mathcal{H} u^{r}(x)\right)^{s / r} .
\end{aligned}
$$

The following theorem will be used in the proof of the main results.

Theorem 2.8 Let $A(t)$ and $W(t)$ be defined as in (20). If $s \geq r>1$, then (here $x \in I$ )

$$
\begin{aligned}
& \frac{1}{W(x)} \int_{a}^{x} \omega(t)\left(\frac{A(t)}{W(t)}\right)^{s} d t \\
& \quad \leq\left(\frac{s}{s-1}\right)^{r} \frac{1}{W(x)} \int_{a}^{x} \omega(t)\left(\frac{A(t)}{W(t)}\right)^{s-r} u^{r}(t) d t-\frac{r}{s-1}\left(\frac{A(x)}{W(x)}\right)^{s} .
\end{aligned}
$$

Proof For any $r \in[0, s]$, we define

$$
\Upsilon_{r}=\frac{1}{W(x)} \int_{a}^{x} \omega(t)\left[\left(\frac{A(t)}{W(t)}\right)^{s-r}\right] u^{r}(t) d t \quad \text { and } \quad \alpha(x)=A(x) / W(x)
$$

Then

$$
\Upsilon_{0}=\frac{1}{W(x)} \int_{a}^{x} \omega(t)\left(\frac{A(t)}{W(t)}\right)^{s} d t, \quad \text { and } \quad \Upsilon_{r}=\frac{1}{W(x)} \int_{a}^{x} \omega(t)\left(\frac{A(t)}{W(t)}\right)^{s-r} u^{r}(t) d t
$$

To prove (28) we need to prove that

$$
\Upsilon_{0} \leq\left(\frac{s}{s-1}\right)^{r} \Upsilon_{r}-\frac{r}{s-1} \alpha^{s}
$$

Now, since $((s-r) / r)+(s(r-1) / r)=s-1$, we can write $\Upsilon_{1}$ as

$$
\Upsilon_{1}=\frac{1}{W(x)} \int_{a}^{x} \omega(t) u(t)\left[\left(\frac{A(t)}{W(t)}\right)^{\frac{s-r}{r}}\right]\left(\frac{A(t)}{W(t)}\right)^{\frac{s(r-1)}{r}} d t
$$


Applying Hölder's inequality, with exponents $r$ and $r /(r-1)$, we get that

$$
\begin{aligned}
\Upsilon_{1} \leq & {\left[\frac{1}{W(x)} \int_{a}^{x} \omega(t) u^{r}(t)\left(\frac{A(t)}{W(t)}\right)^{s-r} d t\right]^{1 / r} } \\
& \times\left[\frac{1}{W(x)} \int_{a}^{x} \omega(t)\left(\frac{A(t)}{W(t)}\right)^{s} d t\right]^{\frac{r-1}{r}} \leq \Upsilon_{r}^{1 / r} \Upsilon_{0}^{(r-1) / r} .
\end{aligned}
$$

Also, from Lemma 2.6 and using the fact that $W(t) \geq \omega(t)$ for $t>a$, we see that

$$
\begin{aligned}
& \frac{1}{W(x)} \int_{a}^{x} \omega(t)\left(\frac{A(t)}{W(t)}\right)^{s} d t \\
& =\frac{s}{s-1} \frac{1}{W(x)} \int_{a}^{x} \omega(s) u(s)\left(\frac{A(t)}{W(t)}\right)^{s-1} d t-\frac{1}{s-1}\left(\frac{A(x)}{W(x)}\right)^{s} \\
& \leq \frac{s}{s-1} \frac{1}{W(x)} \int_{a}^{x} \omega(s) u(s)\left(\frac{A(t)}{W(t)}\right)^{s-1} d t \\
& \quad-\frac{1}{s-1}\left(\frac{A(x)}{W(x)}\right)^{s}=\frac{s}{s-1} \Upsilon_{1}-\frac{1}{s-1} \alpha^{s} .
\end{aligned}
$$

This implies that

$$
\Upsilon_{0} \leq \frac{s}{s-1} \Upsilon_{1}-\frac{1}{s-1} \alpha^{s}
$$

and so

$$
\Upsilon_{1} \geq \frac{s-1}{s} \Upsilon_{0}+\frac{1}{s} \alpha^{s}
$$

Let

$$
\Gamma_{r}:=\Upsilon_{0}-\left(\frac{s}{s-1}\right)^{r} \Upsilon_{r}
$$

Now, using (29) and (30), we get that

$$
\Gamma_{r} \leq \Upsilon_{0}-\left(\frac{s}{s-1}\right)^{r} \frac{\Upsilon_{1}^{r}}{\Upsilon_{0}^{r-1}} \leq \Upsilon_{0}-\left(\frac{s}{s-1}\right)^{r} \Upsilon_{0}^{-r+1}\left(\frac{s-1}{s} \Upsilon_{0}+\frac{1}{s} \alpha^{s}\right)^{r}
$$

Applying Lemma 2.3 with $\rho=\Upsilon_{0}$ and $\rho=\alpha$, we have

$$
\Gamma_{r} \leq-\left(\frac{r}{s-1}\right) \alpha^{s}
$$

which is (28). The proof is complete.

Now, we are in a position to prove the main results. First, we will extend the results due to Bojarski et al. [3] and prove that if $u \in A_{\omega}^{1}(\mathcal{C})$, then $u \in G_{\omega}^{s}(\mathcal{K})$ and give exact values of the new exponents with a sharp constant and exponent similar to the constants in Bojarski et al. [3]. Our method is different from the one used in [3]. 
Theorem 2.9 Assume that $u$ is a nonincreasing weight. If $u \in A_{\omega}^{1}(\mathcal{C})$, i.e.,

$$
\mathcal{H} u(t) \leq \mathcal{C} u(t) \quad \text { for } t \in I, \text { for some } \mathcal{C}>1,
$$

then for $s \in[1, \mathcal{C} /(\mathcal{C}-1))$ we have

$$
\mathcal{H} u^{s}(x) \leq K[\mathcal{H} u(x)]^{s}, \quad \text { where } K=\frac{\mathcal{C}^{1-s}}{\mathcal{C}-s(\mathcal{C}-1)}>0,
$$

and

$$
G_{\omega}^{s}(u) \leq\left(\frac{\mathcal{C}^{1-s}}{\mathcal{C}-s(\mathcal{C}-1)}\right)^{1 /(s-1)}
$$

Proof Let $x \in I$. From the definition of $\mathcal{H} u(t)$ and Lemma 2.6, we see that

$$
\begin{aligned}
\frac{1}{W(x)} \int_{a}^{x} \omega(t)(\mathcal{H} u(t))^{s} d t= & \frac{s}{s-1} \frac{1}{W(x)} \int_{a}^{x} \omega(t) u(t)(\mathcal{H} u(t))^{s-1} d t \\
& -\frac{1}{s-1}(\mathcal{H} u(x))^{s} .
\end{aligned}
$$

This implies that

$$
\frac{1}{W(x)} \int_{a}^{x} \omega(t)\left[u(t)(\mathcal{H} u(t))^{s-1}-\frac{s-1}{s}(\mathcal{H} u(t))^{s}\right] d t=\frac{1}{s}(\mathcal{H} u(x))^{s} .
$$

Let $\Phi(\eta)=\gamma \eta^{s-1}-\frac{s-1}{s} \eta^{s}$ for every $\gamma>0$ and $\eta \geq \gamma$, and we see that

$$
\Phi^{\prime}(\eta)=(s-1) \eta^{s-2}(\gamma-\eta) \leq 0 \text { for } \eta \geq \gamma
$$

so $\Phi(\eta)$ is decreasing for $\eta \geq \gamma$. Fix $t \in(a, x)$. Taking $\gamma=u, \beta=\mathcal{H} u(t)$, and $\delta=\mathcal{C} u(t)$, we have from Lemma 2.2 and condition (32) that $\gamma \leq \beta \leq \delta$, and then

$$
\Phi(\gamma) \geq \Phi(\beta) \geq \Phi(\delta) \quad \text { for } \gamma \leq \beta \leq \delta
$$

Then

$$
\begin{aligned}
u(t)(\mathcal{H} u(t))^{s-1}-\frac{s-1}{s}(\mathcal{H} u(t))^{s} & \geq u(t)(\mathcal{C} u(t))^{s-1}-\frac{s-1}{s}(\mathcal{C} u(t))^{s} \\
& =\mathcal{C}^{s-1} u^{s}(t)-\frac{s-1}{s} \mathcal{C}^{s} u^{s}(t) \\
& =\mathcal{C}^{s-1}\left[1-\frac{s-1}{s} \mathcal{C}\right] u^{s}(t) .
\end{aligned}
$$

From this inequality and (34) we get

$$
\mathcal{C}^{s-1}\left[1-\frac{s-1}{s} \mathcal{C}\right] \frac{1}{W(x)} \int_{a}^{x} \omega(t) u^{s}(t) d t \leq \frac{1}{s}(\mathcal{H} u(x))^{s} .
$$


Thus

$$
\frac{1}{W(x)} \int_{a}^{x} \omega(t) u^{s}(t) d t \leq \frac{\mathcal{C}^{1-s}}{(\mathcal{C}+s-\mathcal{C} s)}(\mathcal{H} u(x))^{s}
$$

which is (33). The proof is complete.

In what follows, we prove the self-improving property of the weighted Gehring class $G_{\omega}^{r}(\mathcal{K})$ with a new constant similar to the constant obtained in [24] for the unweighted class.

Theorem 2.10 Assume that $r>1$ and $u$ is a nonincreasing nonnegative weight. If $u \in$ $G_{\omega}^{r}(\mathcal{K})$, i.e.,

$$
\left(\mathcal{H} u^{r}(t)\right)^{1 / r} \leq \mathcal{K}[\mathcal{H} u(t)] \text { for } t \in I, \text { for some } \mathcal{K}>1
$$

then $u \in G_{\omega}^{s}\left(\mathcal{K}_{1}\right)$ and

$$
\mathcal{H} u^{s}(x) \leq\left(\frac{r}{s} \frac{r-1}{s-1} \mathcal{K}^{r}\right) \frac{1}{\mathcal{K}_{s}}[\mathcal{H} u(x)]^{s} \quad \text { for } x \in I
$$

for every $s \in\left[r, r^{*}\right)$, where $r^{*}$ is the positive root of the equation

$$
\mathcal{K}^{r}\left(\frac{x-r}{x}\right)\left(\frac{x}{x-1}\right)^{r}=1
$$

and $\mathcal{K}_{s}=1-\mathcal{K}^{r} \frac{(s-r)}{s}\left(\frac{s}{s-1}\right)^{r}>0$

Proof Let $x \in I$. From the definition of $\mathcal{H} u(t)$ and Lemma 2.7, we see that

$$
\begin{aligned}
\frac{1}{W(x)} \int_{a}^{x} \omega(t)\left(\mathcal{H} u^{r}(t)\right)^{s / r} d t= & \frac{s}{s-r} \frac{1}{W(x)} \int_{a}^{x} \omega(t) u^{r}(t)\left(\mathcal{H} u^{r}(t)\right)^{\frac{s}{r}-1} d t \\
& -\frac{r}{s-r}\left(\mathcal{H} u^{r}(x)\right)^{s / r} .
\end{aligned}
$$

This implies that

$$
\frac{1}{W(x)} \int_{a}^{x} \omega(t)\left[u^{r}(t)\left(\mathcal{H} u^{r}(t)\right)^{\frac{s}{r}-1}-\frac{s-r}{s}\left(\mathcal{H} u^{r}(t)\right)^{s / r}\right] d t=\frac{r}{s}\left(\mathcal{H} u^{r}(x)\right)^{s / r} .
$$

Let

$$
\Phi(z)=\gamma z^{s / r-1}-\frac{s-r}{s} z^{s / r} \text { for every } \gamma>0 \text { and } z \geq \gamma
$$

and we see (see Theorem 2.9) that $\Phi$ is decreasing. Fix $t \in(a, x)$. Now let

$$
\beta=\mathcal{H} u^{r}(t), \quad \gamma=u^{r} \text { and } \delta=(\mathcal{K} \mathcal{H} u(t))^{r} .
$$

It is clear from (35) that $\gamma \leq \beta \leq \delta$ and

$$
\Phi(\gamma) \geq \Phi(\beta) \geq \Phi(\delta) \text { for } \gamma \leq \beta \leq \delta,
$$


so

$$
\begin{aligned}
\Phi\left(\mathcal{H} u^{r}(t)\right) & =\gamma\left(\mathcal{H} u^{r}(t)\right)^{s / r-1}-\frac{s-r}{s}\left(\mathcal{H} u^{r}(t)\right)^{s / r} \\
& \geq u^{r}\left((\mathcal{K} \mathcal{H} u(t))^{r}\right)^{s / r-1}-\frac{s-r}{s}\left((\mathcal{K} \mathcal{H} u(t))^{r}\right)^{s / r} .
\end{aligned}
$$

This and (37) imply that

$$
\begin{aligned}
& \frac{1}{W(x)} \int_{a}^{x} \omega(t)\left[u^{r}(t)\left((\mathcal{K} \mathcal{H} u(t))^{r}\right)^{s / r-1}-\frac{s-r}{s}\left((\mathcal{K H} u(t))^{r}\right)^{s / r}\right] d t \\
& \quad \leq \frac{r}{s}\left(\mathcal{H} u^{r}(x)\right)^{s / r}
\end{aligned}
$$

and so

$$
\begin{aligned}
& \frac{1}{W(x)} \int_{a}^{x} \omega(t)\left[u^{r}(t) \mathcal{K}^{s-r}(\mathcal{H} u(t))^{s-r}-\frac{s-r}{s} \mathcal{K}^{s}(\mathcal{H} u(t))^{s}\right] d t \\
& \quad \leq \frac{r}{s}\left(\mathcal{H} u^{r}(x)\right)^{s / r} .
\end{aligned}
$$

Now, since $\mathcal{H} u^{r}(t) \leq \mathcal{K}^{r}[\mathcal{H} u(t)]^{r}$, we get that

$$
\begin{aligned}
& \frac{1}{W(x)} \int_{a}^{x} \omega(t)\left[u^{r} \mathcal{K}^{s-r}(\mathcal{H} u(t))^{s-r}-\frac{s-r}{s} \mathcal{K}^{s}(\mathcal{H} u(t))^{s}\right] d t \\
& \leq \frac{r}{s} \mathcal{K}^{s}[\mathcal{H} u(x)]^{s} .
\end{aligned}
$$

Thus

$$
\begin{aligned}
& \frac{1}{W(x)} \int_{a}^{x} \omega(t) u^{r}(t)(\mathcal{H} u(t))^{s-r} d t \\
& \quad \leq\left(\frac{s-r}{s}\right) \frac{\mathcal{K}^{r}}{W(x)} \int_{a}^{x} \omega(t)(\mathcal{H} u(t))^{s} d t+\frac{r}{s} \mathcal{K}^{r}[\mathcal{H} u(x)]^{s}
\end{aligned}
$$

Now, from Theorem 2.8, we see that

$$
\begin{aligned}
& \frac{1}{W(x)} \int_{a}^{x} \omega(t)(\mathcal{H} u(t))^{s} d t \\
& \quad \leq\left(\frac{s}{s-1}\right)^{r} \frac{1}{W(x)} \int_{a}^{x} \omega(t)(\mathcal{H} u(t))^{s-r}(u(t))^{r} d t-\frac{r}{s-1}(\mathcal{H} u(x))^{s} \\
& \quad \leq\left(\frac{s}{s-1}\right)^{r} \frac{1}{W(x)} \int_{a}^{x} \omega(t)(\mathcal{H} u(t))^{s-r} u^{r}(t) d t-\frac{r}{s-1}(\mathcal{H} u(x))^{s} .
\end{aligned}
$$

This and (38) imply that

$$
\begin{aligned}
& \frac{1}{W(x)} \int_{a}^{x} \omega(t) u^{r}(t)(\mathcal{H} u(t))^{s-r} d t \\
& \leq\left(\frac{s-r}{s}\right)\left(\frac{s}{s-1}\right)^{r} \frac{\mathcal{K}^{r}}{W(x)} \int_{a}^{x} \omega(t)(\mathcal{H} u(t))^{s-r}(u(t))^{r} d t \\
& \quad+\left(\frac{r r-1}{s-1} \mathcal{K}^{r}\right)[\mathcal{H} u(x)]^{s} .
\end{aligned}
$$


Then

$$
\begin{aligned}
& {\left[1-\mathcal{K}^{r}\left(\frac{s-r}{s}\right)\left(\frac{s}{s-1}\right)^{r}\right] \frac{1}{W(x)} \int_{a}^{x} \omega(t) u^{r}(t)(\mathcal{H} u(t))^{s-r} d t} \\
& \quad \leq\left(\frac{r}{\left.s-\frac{r-1}{s-1} \mathcal{K}^{r}\right)[\mathcal{H} u(x)]^{s} .}\right.
\end{aligned}
$$

Since $\mathcal{H} u^{r} \geq u^{r}$, where $u$ is nonincreasing, we obtain

$$
\left[1-\mathcal{K}^{r}\left(\frac{s-r}{s}\right)\left(\frac{s}{s-1}\right)^{r}\right] \frac{1}{W(x)} \int_{a}^{x} \omega(t) u^{s}(t) d t \leq\left(\frac{r}{s-1} \frac{r-1}{s-1}\right)[\mathcal{H} u(x)]^{s}
$$

Let

$$
\psi_{r}(x)=1-\mathcal{K}^{r} \phi(x, r)
$$

where

$$
\phi(x, r)=\left(\frac{x-r}{x}\right)\left(\frac{x}{x-1}\right)^{r} .
$$

Clearly $\psi_{r}(r)=1>0$, and as $-\mathcal{K}^{r} \phi(x, r)$ is a strictly decreasing function for positive values of $x$, the same holds for $\psi_{r}(x)$ which will be zero for a certain value $r^{*}>r$ given by the unique positive solution of the equation $\mathcal{K}^{r} \phi(x, r)=1$. Then $\psi_{r}\left(r^{*}\right)=0$ and

$$
\psi_{r}(x)>0 \quad \Leftrightarrow \quad \mathcal{K}^{r} \phi(x, r)<1 .
$$

Thus we have that $\psi_{r}(x)>0$ in $\left[r, r^{*}\right)$, and from (39) we obtain

$$
\mathcal{H} u^{s}(x) \leq\left(\frac{r}{s} \frac{r-1}{s-1} \mathcal{K}^{r}\right) \frac{1}{\psi_{r}(s)}[\mathcal{H} u(x)]^{s} \quad \text { for } s \in\left[r, r^{*}\right)
$$

which is (36). The proof is complete.

Remark 2.3 We mention here that the result given in the literature for the self-improving property of the Gehring class is

$$
\left[G^{s}(u)\right]^{1 / s^{\prime}} \leq \mathcal{K}^{1 / r^{\prime}}\left(\frac{r}{s \mathcal{K}(s)}\right)^{1 / r}
$$

whereas our result is

$$
\left[G_{\omega}^{s}(u)\right]^{1 / s^{\prime}} \leq\left[\frac{r r-1}{s} \frac{\mathcal{K}^{r}}{s-1}\right]^{1 / s}
$$

\section{Applications}

The properties of the nonincreasing rearrangement and the relations between $\vartheta$ and $\vartheta^{*}$ which are proved in $[9,15]$ play important roles in extending the results to the $n$ dimensional case. As usual, we assume that $r_{0}$ is any cube in $\mathbb{R}^{n}$ and by $\left|r_{0}\right|$ we mean its Lebesgue measure. Let $\Phi$ be the class of positive convex functions, and let us denote 
with $\vartheta_{*}$ and $\vartheta^{*}$ respectively the nonincreasing and nondecreasing rearrangements for the function $\vartheta$. The functions $\vartheta_{*}$ and $\vartheta^{*}$ are equimeasurable with $\vartheta$ in a set $r_{0}$ in the sense that for any real exponent $r$ the following holds:

$$
\int_{r_{0}} \vartheta^{r}(x) d x=\int_{0}^{\left|r_{0}\right|}\left(\vartheta^{*}(s)\right)^{r} d s=\int_{0}^{\left|r_{0}\right|}\left(\vartheta_{*}(s)\right)^{r} d s \quad \text { for all } r>1
$$

It is well known that a convex function $\varphi \in \Phi$ verifies the so-called Jensen inequality

$$
\varphi\left(\frac{1}{\left|r_{0}\right|} \int_{r_{0}} \vartheta(x) d x\right) \leq \frac{1}{\left|r_{0}\right|} \int_{r_{0}} \varphi(\vartheta(x)) d x
$$

This makes natural to define that a weight $\vartheta$ is said to verify the reverse Jensen inequality that will be denoted by $\vartheta \in \mathcal{J}_{\varphi}(K)$ if there exists a real constant $\mathcal{K}>1$ such that for every bounded interval $I$ the following holds:

$$
\frac{1}{|I|} \int_{I} \varphi(\vartheta(x)) d x \leq \mathcal{K} \varphi\left(\frac{1}{|I|} \int_{I} \vartheta(x) d x\right)
$$

The following theorem, proved by Korenovskii in [15], provides the exact estimate for the equimeasurable rearrangements of weights verifying the reverse Jensen inequality.

Theorem 3.1 Let $\varphi \in \Phi$ and $\vartheta \in \mathcal{J}_{\varphi}(\mathcal{K})$. Then

$$
\frac{1}{\left|r_{0}\right|} \int_{r_{0}} \varphi\left(\vartheta^{*}(x)\right) d x \leq \mathcal{K} \varphi\left(\frac{1}{\left|r_{0}\right|} \int_{r_{0}} \vartheta^{*}(x) d x\right)
$$

with the same constant $\mathcal{K}$ as in condition (40).

It is easy to observe that, for $\varphi(u)=u^{r}$ or $\varphi(u)=u^{\frac{1}{1-s}}$, the reverse Jensen inequality corresponds respectively to the $G^{r}$ and the $A^{s}$ conditions. Similarly, for $\varphi(u)=u^{s / r}$, it becomes the $G^{s, r}$ condition. This means that Theorems 2.9, 2.10, 3.1 will allow us to extend our proofs to obtain the same results in the general $n$-dimension. This will be obtained by using the properties of the measure on the space and studying the reverse of Jensen's inequality with a weight of the form

$$
\varphi\left(\frac{1}{H(t)} \int_{a}^{t}|h(s)| g(s) d s\right) \leq \frac{1}{H(t)} \int_{a}^{t}|h(s)| \varphi(g(s)) d s,
$$

where $H(t)=\int_{a}^{t}|h(s)| d s$. On the other hand, the self-improving property has applications in different fields, for example, in higher integrability theory and in optimal regularity of solutions to some elliptic $s D E s$, see Kenig [14]) and Martio and Sbordone [20]. In the following, we apply our results to prove a higher integrability theorem. Note that, for all nonnegative and nonincreasing functions $u$ and $r>1$, we have

$$
\begin{aligned}
\mathcal{H} u^{r}(t) & =\frac{1}{W(t)} \int_{0}^{t} \omega(s) u^{r}(s) d s=\frac{1}{W(t)} \int_{0}^{t} \omega(s) u^{r-1}(s) u(s) d s \\
& \geq \frac{u^{r-1}(t)}{W(t)} \int_{0}^{t} \omega(s) u(s) d s \quad \text { for all } t \in I .
\end{aligned}
$$


Consider the class of nonnegative and nonincreasing functions $u$ that satisfy the reverse of (42) in terms of $\mathcal{H}$, namely

$$
\mathcal{H} u^{r}(t) \leq \mathcal{K} u^{r-1}(t) \mathcal{H} u(t) \quad \text { for all } t \in I \text { and } \mathcal{K}>1 \text {. }
$$

Theorem 3.2 Assume that $u$ is a nonincreasing weight such that (43) holds for some $\mathcal{K}>1$. If $r>1$ and $\mathcal{K} r>r-1$, then for $s \in\left[r, r \mathcal{K}_{r} /\left(\mathcal{K}_{r}-1\right)\right)$, where

$$
\mathcal{K}_{r}=\frac{r \mathcal{K}}{r-1}
$$

we have

$$
\mathcal{H} u^{s}(t) \leq K\left[\mathcal{H} u^{r}(t)\right]^{s / r} \quad \text { for } t \in I \text {, where } K:=\frac{\mathcal{K}_{r}^{1-r+(s / r)}}{\mathcal{K}_{r}-\frac{s}{r}\left(\mathcal{K}_{r}-1\right)}
$$

Proof Let $t \in I$ and $F(t)=\mathcal{H} u^{r}(t)$. Using Hölder's inequality with exponents $r$ and $r /(r-1)$ and (43), we obtain

$$
\begin{aligned}
\frac{1}{W(t)} \int_{0}^{t} \omega(s) F(s) d s \leq & \frac{\mathcal{K}}{W(t)} \int_{0}^{t} u^{r-1}(s) \frac{\omega(s)}{W(s)} \int_{0}^{s} \omega(\tau) u(\tau) d \tau d s \\
\leq & \mathcal{K}\left[\frac{1}{W(t)} \int_{0}^{t} \omega(s) u^{r}(s) d s\right]^{(r-1) / r} \\
& \times\left[\frac{1}{W(t)} \int_{0}^{t} \omega(s)\left(\frac{1}{W(s)} \int_{0}^{s} \omega(\tau) u(\tau) d \tau\right)^{r} d s\right]^{1 / r} .
\end{aligned}
$$

Using the definition of $\mathcal{H} u$, we get that

$$
\mathcal{H} F(t)=\frac{1}{W(t)} \int_{0}^{t} \omega(s) F(s) d s \leq \mathcal{K}\left[\mathcal{H} u^{r}(t)\right]^{(r-1) / r}\left[\mathcal{H}[\mathcal{H} u(t)]^{r}\right]^{1 / r} .
$$

From Lemma 2.5, we see that

$$
\left[\mathcal{H}[\mathcal{H} u(t)]^{r}\right]^{1 / r} \leq \frac{r}{r-1}\left[\mathcal{H} u^{r}(t)\right]^{1 / r} .
$$

Combining (46) and (47), we have that

$$
\begin{aligned}
\mathcal{H} F(t) & =\frac{1}{W(t)} \int_{0}^{t} \omega(s) F(s) d s \leq \frac{r \mathcal{K}}{(r-1)}\left[\mathcal{H} u^{r}(t)\right]^{(r-1) / r}\left[\mathcal{H} u^{r}(t)\right]^{1 / r} \\
& =\frac{r \mathcal{K}}{(r-1)} \mathcal{H} u^{r}(t)=\mathcal{K}_{r} \mathcal{H} u^{r}(t)=\mathcal{K}_{r} F(t),
\end{aligned}
$$

i.e.,

$$
\mathcal{H} F(t) \leq \mathcal{K}_{r} F(t)
$$

note $\mathcal{K}_{r}>1$ since $r \mathcal{K}>r-1$. Since $F$ is nonnegative and nonincreasing (see Remark 2.2), the assumptions of Theorem 2.9 are satisfied (with $u$ replaced with $F$; see (48)), so

$$
\mathcal{H} F^{r}(t) \leq A[\mathcal{H} F(t)]^{r}
$$


with $A=\mathcal{K}_{r}^{1-r} /\left(\mathcal{K}_{r}-r\left(\mathcal{K}_{r}-1\right)\right)$ for $r=s / r \in\left[1, \mathcal{K}_{r} /\left(\mathcal{K}_{r}-1\right)\right)$. Note

$$
F(t)=(1 / W(t)) \int_{0}^{t} \omega(s) u^{r}(s) d s \geq u^{r}(t),
$$

so this together with (48) and (49) yields

$$
\begin{aligned}
\mathcal{H} u^{s}(t) & =\frac{1}{W(t)} \int_{0}^{t} \omega(s)\left(u^{r}(s)\right)^{r} d s \leq \frac{1}{W(t)} \int_{0}^{t} \omega(s)(F(s))^{r} d s \\
& =\mathcal{H} F^{r}(t) \leq A[\mathcal{H} F(t)]^{r} \leq A \mathcal{K}_{r}^{r}[F(t)]^{r} \\
& =K[F(t)]^{r}=K\left[\mathcal{H} u^{r}(t)\right]^{s / r},
\end{aligned}
$$

which proves (44). The proof is complete.

Now, we show that the self-improving property of the weighted Gehring class can be applied to prove the self-improving property of the Muckenhoupt class with a sharp value of the exponents. Assume $u \in A^{s}(\mathcal{C})$, i.e., the condition

$$
\left(\frac{1}{|I|} \int_{I} u(t) d t\right)\left(\frac{1}{|I|} \int_{I} u^{-\frac{1}{s-1}}(t) d t\right)^{s-1} \leq \mathcal{C}
$$

holds. This condition can be rewritten in the form

$$
\left(\frac{1}{\Omega(I)} \int_{I}\left(\frac{1}{u(s)}\right)^{s^{*}} u(s) d s\right)^{\frac{1}{s^{*}}} \leq \mathcal{C}^{\left(\frac{s^{*}-1}{s^{*}}\right)}\left(\frac{1}{\Omega(I)} \int_{I}\left(\frac{1}{u(s)}\right) u(s) d s\right)
$$

where $\Omega(I)=\int_{I} u(t) d t$ and $s^{*}=s /(s-1)$. From this condition, we see that $u \in A^{s}(\mathcal{C})$ if and only if $u^{-1} \in G_{u}^{r}(\mathcal{K})$ with $\mathcal{K}=\mathcal{C}^{\frac{s^{*}-1}{s^{*}}}$ and $r=s^{*}$. From Theorem 2.10 inequality (51) holds for every $s \in\left[r, s^{*}\right)$, where $s^{*}$ is the solution of the equation

$$
1-\left(\frac{x-r}{x}\right)\left(\frac{\mathcal{K} x}{x-1}\right)^{r}=0 .
$$

Using the value of $r=s^{*}$ and $\mathcal{K}=\mathcal{C}^{\frac{s^{*}-1}{s^{*}}}$, we have

$$
\left(\frac{x-s^{*}}{x}\right)\left(\frac{\mathcal{C}^{\frac{s^{*}-1}{s^{*}} x}}{x-1}\right)^{s^{*}}=1
$$

Since $s^{*}=s /(s-1)$, by rewriting the last equation in terms of $s$, we see that $s^{*}$ is given from the solution of the equation

$$
\left(\frac{(s-1) x-s}{(s-1) x}\right)\left(\frac{\mathcal{K}^{1 / s} x}{x-1}\right)^{\frac{1}{s-1}}=1 .
$$

Using the transformation $x \rightarrow x /(x-1)$, we then get $r_{*}\left(r^{*}=s^{*} /\left(s^{*}-1\right)\right)$ is given from the solution of the equation

$$
\left(\frac{s-x}{s-1}\right)(\mathcal{C} x)^{\frac{1}{s-1}}=1,
$$

which is the same condition as in Korenovskii [15]. 
The self-improving property has applications in different fields especially in studying the optimal regularity of solutions to some elliptic $S D E s$ (see for example Kenig [14]) where the $L^{s}$ solvability of the Dirichlet problem $\operatorname{div} A(x) \nabla u=0$ on the unit disc $D$, with $\left.u\right|_{D}=\varphi$, can be expressed in terms of $G^{r}$ conditions on the boundary $\partial D$ for the harmonic measures associated with $A(x)$, with $1 / s+1 / r=1$.

Another application of the sharp results for the reverse Hölder inequalities can be found in Martio and Sbordone [20] in the study of $K$-quasiminimizers and their inverse. For other applications of inequalities including extrapolation theory, vector-valued inequalities, and estimate for certain classes of nonlinear partial differential equations, we refer the reader to the book [14]. In the following, we report an application of the self-improving property in the solvability of the Neumann problem for divergence form elliptic operators and $L^{s}$ data in the half plane by using the relation between $G^{s}$ and $A^{s}$ that has been proved by Johnson and Neugebauer [13]. We begin reporting a result contained in [13] that shows the $A^{s}$-regularity of the derivative of a homeomorphism of the real line and the derivative of its inverse.

Theorem 3.3 Let $h: \mathbb{R}_{+} \rightarrow \mathbb{R}_{+}$be an increasing homeomorphism onto such that $h, h^{-1}$ are locally absolutely continuous. Then

$$
h^{\prime} \in A^{s} \quad \Leftrightarrow \quad\left(h^{-1}\right)^{\prime} \in G^{r}, \quad \frac{1}{s}+\frac{1}{r}=1,
$$

and the constants $A^{s}\left(h^{\prime}\right)$ and $G^{r}\left(\left(h^{-1}\right)^{\prime}\right)$ are equal.

Now we show that Theorem 3.3 for regularity and the self-improving Theorem 2.10 can be applied to the solvability of Neumann problems for divergence elliptic operators with $L^{s}$ data (see [5]). Let us consider the following Neumann problem:

$$
\Delta \vartheta=0 \quad \text { in } \mathbb{R}_{+}^{2} \text { and }\left.\quad \frac{\partial \vartheta}{\partial t}\right|_{\mathbb{R}_{+}}=f
$$

Let us consider a quasiconformal mapping $\Phi: \mathbb{R}_{+}^{2} \rightarrow \mathbb{R}_{+}^{2}$ with $\Phi(x, 0)=h(x)$, where $h$ : $\mathbb{R}_{+} \rightarrow \mathbb{R}_{+}$is a homeomorphism, and let us consider the pull-back Laplacian matrix

$$
A(x, t)=\left(D \Phi^{t}\right)^{-1}|D \Phi|\left(D \Phi^{t}\right)^{-1}
$$

If the solution of (52) is composed with $\Phi$, we have that $u=\vartheta \circ \Phi$ is a solution of the following Neumann problem:

$$
\operatorname{div} A \nabla u=0 \quad \text { in } \mathbb{R}_{+}^{2} \quad \text { and }\left.\quad A \nabla u \cdot \vec{N}\right|_{\mathbb{R}}=(f \circ h) h^{\prime}
$$

By using the variational formulation of the Neumann problem and assuming that $d h=$ $h^{\prime} d x$, we can see that the Neumann data for $u=\vartheta \circ \Phi$ are $(f \circ h) h^{\prime}$. For this to belong to $L^{s}$, we need that $\int|(f \circ h)|^{s}\left|h^{\prime}\right|^{s}<\infty$, which is equivalent, if $y=h(x), d y=h^{\prime}(x) d x$, to the fact that $f \in L_{u}^{s}$, where $u(y)=\left|h^{\prime}\left(h^{-1}(y)\right)\right|^{s-1}$. Now, if (53) was solvable for $L$, then all derivatives of $u$ restricted to $\mathbb{R}$ would be in $L^{s}$. This implies, in particular, that if in (53) $f \in L^{s}(u d x)$, 
$\frac{\partial \vartheta}{\partial y}=H(y) \in L_{u}^{s}$, where $H(f)$ is the classical Hilbert transform

$$
H f(y)=\lim _{\epsilon \rightarrow 0} \frac{1}{\pi} \int_{|x-y|>\epsilon} \frac{f(x)}{x-y} d y,
$$

and the bound

$$
\|H f\|_{L_{u}^{s}} \leq \mathcal{C}\|f\|_{L_{u}^{s}}
$$

holds. But it is well known that (54) holds if and only if $u \in A^{s}(\mathcal{C})$ (see [12]). But $u \in A^{s}(\mathcal{C})$ is equivalent to $\left(h^{-1}\right)^{\prime} \in A^{r}\left(\mathcal{C}_{r}\right)$ with $r=s /(s-1)$. Now, by Theorem 3.3 , this is equivalent to $h^{\prime} \in G^{s}(\mathcal{K})$ for any bounded interval $I$ of $\mathbb{R}^{+}$with a constant $\mathcal{K}=G^{s}\left(h^{\prime}\right)$. Applying Theorem 2.10, we see that if $s^{*}$ is the unique positive root of the equation $\omega(x, s)=\mathcal{K}$, then we have $h^{\prime} \in G^{s}\left(\mathcal{K}_{s}\right)$ for every $s \in\left[s, s^{*}\right)$ and

$$
\mathcal{M}\left(h^{\prime}(t)\right)^{s} \leq \mathcal{K}_{s}\left[\mathcal{M}\left(h^{\prime}(t)\right)\right]^{s}, \quad \mathcal{K}_{s}=\frac{s}{s \mathcal{K}}
$$

is a positive root of $\omega(s, s)=\mathcal{K}$.

\section{Acknowledgements}

The second author is highly thankful to Prince Sultan University and OSTIM Technical University for supporting this work.

Funding

None.

Availability of data and materials

None.

Competing interests

The authors declare that they have no competing interests.

\section{Authors' contributions}

All authors have taken equal part in this research and they read and approved the final manuscript.

\section{Author details}

${ }^{1}$ Department of Mathematics, Faculty of Science, Mansoura University, 35516 Mansoura, Egypt. ${ }^{2}$ Department of Mathematics, Faculty of Science, New Mansoura University, New Mansoura City, Egypt. ${ }^{3}$ Department of Mathematics and General Sciences, Prince Sultan University, 11586 Riyadh, Saudi Arabia. ${ }^{4}$ Department of Industrial Engineering, OSTiM Technical University, 06374 Ankara, Turkey. ${ }^{5}$ School of Mathematics, Statistics and Applied Mathematics, National University of Ireland, Galway, Ireland. ${ }^{6}$ Department of Mathematics, Texas A \& M University-Kingsville, Kingsville, Texas 78363, USA.

\section{Publisher's Note}

Springer Nature remains neutral with regard to jurisdictional claims in published maps and institutional affiliations.

Received: 12 April 2021 Accepted: 5 August 2021 Published online: 26 August 2021

\section{References}

1. Agarwal, R.P., O'Regan, D., Saker, S.H.: Self-improving properties of a generalized Muckenhoupt class. Acta Math. Hung. 164, 113-134 (2021)

2. Bojarski, B.: Remarks on the stability of reverse Hölder inequalities and quasiconformal mappings. Ann. Acad. Sci. Fenn., Ser. A 1 Math. 10, 89-94 (1985)

3. Bojarski, B., Sbordone, C., Wik, I.: The Muckenhoupt class $A_{1}(R)$. Stud. Math. 101, 155-163 (1992)

4. Coifman, R.R., Fefferman, C.: Weighted norm inequalities for maximal functions and singular integrals. Stud. Math. 51, 241-250 (1974)

5. Corporente, R.: Weighted integral inequalities, BMO-spaces and applications. Ph.D. dissertation (2007)

6. D’Apuzzo, L., Sbordone, C.: Reverse Hölder inequalities, a sharp result. Rend. Mat. Appl. (7) 10, 357-366 (1990)

7. Dindoš, M., Wall, T.: The sharp $A^{\text {s }}$ constant for weights in a reverse Hölder class. Rev. Mat. Iberoam. 25(2), 559-594 (2009)

8. Franciosi, M.: Weighted rearrangement and higher integrability results. Stud. Math. 92, 31-39 (1989) 
9. Franciosi, M., Moscariello, G.: Higher integrability results. Manuscr. Math. 52, 151-170 (1985)

10. Gehring, F.W.: The $L^{5}$-integrability of the partial derivatives of a quasiconformal mapping. Bull. Am. Math. Soc. 97, 465-466 (1973)

11. Gehring, F.W.: The $L^{5}$-integrability of the partial derivatives of a quasi-conformal mapping. Acta Math. 130, 265-277 (1973)

12. Hunt, R., Muckenhoupt, B., Wheeden, R.: Weighted norm inequalities for the conjugate function and Hilbert transform. Trans. Am. Math. Soc. 176, 227-251 (1973)

13. Johnson, R., Neugebauer, C.J.: Homeomorphisms preserving Ás. Rev. Mat. Iberoam. 3, 249-273 (1987)

14. Kenig, C.E.: Harmonic Analysis Techniques for Second Order Elliptic Boundary Value Problems. CBMS Regional Conf. Series in Math., vol. 83, xii + 146 pp. Am. Math. Soc., Providence (1994)

15. Korenovskii, A.A.: The exact continuation of a reverse Hölder inequality and Muckenhoupt's conditions. Math. Notes 52, 1192-1201 (1992)

16. Korenovskii, A.A.: Mean Oscillations and Equimeasurable Rearrangements of Functions. Lecture Notes of the Unione Matematica Italiana, vol. 4. Springer, Berlin; UMI, Bologna (2007)

17. Korenovskii, A.A., Lerner, A.K., Stokolos, A.M.: A note on the Gurov-Reshetnyak condition. Math. Res. Lett. 9, 579-585 (2002)

18. Malaksiano, N.A.: The exact inclusions of Gehring classes in Muckenhoupt classes. Mat. Zametki 70(5), 742-750 (2001) (Russian), translation in Math. Notes 70, 673-681 (2001)

19. Malaksiano, N.A.: The precise embeddings of one-dimensional Muckenhoupt classes in Gehring classes. Acta Sci. Math. 68, 237-248 (2002)

20. Martio, O., Sbordone, C.: Quasiminimizers in one dimension: integrability of the derivative, inverse function and obstacle problems. Ann. Mat. Pura Appl. 186, 579-590 (2007)

21. Muckenhoupt, B.: Weighted norm inequalities for the Hardy maximal function. Trans. Am. Math. Soc. 165, $207-226$ (1972)

22. Nikolidakis, E.N.: $A_{1}$-weights on $\mathbb{R}$ : an alternative approach. arXiv:1312.1742v2

23. Nikolidakis, E.N.: A Hardy inequality and applications to reverse Hölder inequalities for weights on $\mathbb{R}$. arXiv:1312.1991v3

24. Nikolidakis, E.N., Stavropoulos, T.: A refinement of a Hardy type inequality for negative exponents, and sharp applications to Muckenhoupt weights on $\mathbb{R}$. arXiv:1804.00840v3

25. Pérez, C.: Weighted norm inequalities for general maximal operators. Publ. Math. 35, 169-186 (1991)

26. Popoli, A.: Weighted reverse Hölder inequalities. Rend. Accad. Sci. Fis. Mat. 62, 187-211 (1995)

27. Popoli, A.: Sharp integrability exponents and constants for Muckenhoupt and Gehring weights as solutions to a unique equation. Ann. Acad. Sci. Fenn., Math. 43, 785-805 (2018)

28. Saker, S.H., O'Regan, D., Agarwal, R.P.: A higher integrability theorem from a reverse weighted inequality. Bull. Lond. Math. Soc. 51, 967-977 (2019)

29. Saker, S.H., O'Regan, D., Osman, M.M., Agarwal, R.P.: New dynamic inequalities for decreasing functions and theorems of higher integrability. Ann. Appl. Math. 42, 1-13 (2018)

30. Sbordone, S.: Higher integrability from reverse integral inequalities, methods of functional analysis and the theory of elliptic equations, Napoli 1982, pp. 347-357 (1982)

31. Sbordone, S.: Some reverse integral inequalities. Atti Accad. Pontaniana, Napoli (1984)

32. Sbordone, S.: Rearrangement of functions and reverse Hölder inequalities. In: Ennio De Giorgi colloquium (Paris, 1983). Res. Notes in Math., vol. 125, pp. 139-148. Pitman, Boston (1985)

33. Vasyunin, V.: The exact constant in the inverse Hölder inequality for Muckenhoupt weights. Algebra Anal. 15(1), 73-117 (2003) (Russian), translation in St. Petersburg Math. J. 15(1), 49-79 (2004)

\section{Submit your manuscript to a SpringerOpen ${ }^{\circ}$ journal and benefit from:}

- Convenient online submission

- Rigorous peer review

- Open access: articles freely available online

- High visibility within the field

- Retaining the copyright to your article

Submit your next manuscript at $\boldsymbol{s p r i n g e r o p e n . c o m ~}$ 